\title{
Repercusiones de la sociedad posmoderna en el ciclo de vida familiar. La emancipación de los hijos del hogar: una transición "interminable".
}

\author{
Alicia Bonelli ${ }^{1}$ \\ Universidad Argentina de la Empresa
}

\section{Tipo de trabajo: Artículo}

Material original autorizado para su primer publicación en el Journal de Ciencias Sociales, Revista Académica de la Facultad de Ciencias Sociales de la Universidad de Palermo.

Recibido: 19-4-2014

Aceptado: 5-8-2014

\section{Resumen}

En los últimos años hemos asistido a la emergencia, en el seno de algunas familias nucleares de clase media o superior con hijos en su mayoría universitarios del Área Metropolitana de la Ciudad de Buenos Aires, de un fenómeno denominado "nido lleno". Éste consiste en la permanencia de los hijos adultos jóvenes en la familia de origen por tiempo prolongado. Esta problemática también es conocida como síndrome de Peter Pan, haciendo referencia a la condición de "eterna" adolescencia de los jóvenes que habitan este estilo de configuración familiar.

El objetivo de este trabajo se circunscribe, dada la existencia de estructuras y arreglos familiares diversos, a comprender de qué manera la sociedad posmoderna impacta, exclusivamente, en el ciclo de vida en este tipo de familias y más precisamente en el proceso de emancipación de sus hijos.

El abordaje de este fenómeno se realizará desde el marco de la psicología social construccionista. Como conclusión se sostiene que este estilo de familia se caracteriza por la conjunción de lo viejo y lo nuevo y por la transformación permanente. Hoy uno de sus cambios es la extensión temporal de la etapa del proceso de emancipación de los hijos del hogar. Esto se explica por la confluencia de diversos ideales en el seno de la misma. Por un lado estos padres sostienen a sus hijos, quienes les otorgan sentido a sus propias vidas, y así evitan encontrarse con el vacío que implica la ausencia de un proyecto propio (nido vacío). Por otro lado, los hijos buscan construir su identidad personal a través del consumo, lo cual se torna contradictorio con el diseño de un plan a futuro que les permita el logro de la autonomía económica. Asimismo, no quieren enfrentarse a una realidad sociocultural que promete un desafío para el cual no están preparados y que tampoco les asegura ventaja alguna en lo que respecta a la inserción laboral, pese a contar con estudios universitarios. Esta prolongación representa una amenaza para el logro de la identidad de estos jóvenes.

Palabras claves: posmodernidad; familia; emancipación de los jóvenes; ideales

\footnotetext{
${ }^{1}$ Docente Universidad Argentina de la Empresa- UADE. E.mail: aliciaboelli@hotmail.com
} 


\section{Abstract}

In recent years we have witnessed the emergence, within some nuclear families of middle or upper class children mostly university students from de Metropolitan Area of Buenos Aires, a phenomenon called "full fledge". It is the retention of young adult children in family home for long time. This problem is also known as Peter Pan syndrome, referring to the status of "eternal" adolescence young people who inhabit this family-style setting. The objective of this work is limited, given the existence of various family structures and arrangements, to understand how postmodern society hits exclusively on the life cycle in such families and more precisely in the emancipation of their children. Dealing with this phenomenon will be from within the social constructionist psychology. In conclusion it is argued that this style of family is characterized by the combination of the old and the new and permanent transformation. Today one of the changes is the temporary extension step process of emancipation of the children of the household. This is explained by the confluence of different ideals within it. On the one hand these parents support their children, who give them meaning to their own lives, and thus avoid encountering the gap implies the absence of an independent project (empty nest). On the other hand the children, looking to build their personal identity through consumption, which becomes inconsistent with the design of a future plan that allows them to achieve economic autonomy. Also, they do not want to face a sociocultural reality that promises a challenge for which they are not prepared, nor assures advantage in regard to the employment despite having college. This extension represents a threat to the achievement of the identity of these young people.

Keywords: postmodernism, family, youth empowerment, ideal.

\section{Introducción}

En los últimos años hemos asistido a la emergencia, en el seno de algunas familias nucleares de clase media o superior con hijos en su mayoría universitarios del Área Metropolitana de la Ciudad de Buenos Aires, de un fenómeno denominado "nido lleno". Éste consiste en la permanencia de los hijos adultos jóvenes en la familia de origen por tiempo prolongado. Esta problemática también es conocida como síndrome de Peter Pan, haciendo referencia a la condición de "eterna" adolescencia de estos jóvenes.

En algunos casos se trata de hijos que dependen económicamente de los padres a pesar de tener sus propios ingresos, debido a que los destinan exclusivamente a satisfacer sus gustos personales, en otros casos no trabajan y la provisión de recursos por parte de los adultos es total y también están aquellos que, si bien en algún momento dejaron el hogar paterno, por circunstancias como divorcios o reducción de recursos económicos, retornan al "nido" en el cual vuelven para encontrar el sostén de los adultos.

El abordaje de este fenómeno se realizará desde el marco de la psicología social construccionista considerando especialmente los desarrollos referidos a la temática de familia y a las etapas normativas del ciclo de vida del tipo de familia al que este trabajo hace referencia.

En relación a esto último, Duvall y Rodgers (citados en Gimeno Collado, 1999, p.113) consideran tres 
etapas en el ciclo de vida familiar: etapa de constitución (elección de pareja y noviazgo, matrimonio y cohabitación, parejas jóvenes sin hijos); etapa de expansión (transición a la paternidad, familias con hijos en edad escolar, familias con hijos adolescentes) y etapa de reducción (familias con hijos en proceso de emancipación, familias con el nido vacío, familias retiradas)

El objetivo de este ensayo se circunscribe, dada la existencia de estructuras y arreglos familiares diversos, a reflexionar acerca de la manera en que la sociedad posmoderna impacta, exclusivamente, en el ciclo de vida en este tipo de familia y más precisamente en la postergación del proceso de emancipación de sus hijos. Al hacer referencia a la sociedad posmoderna se tendrán en cuenta los siguientes factores del contexto: la práctica del consumismo, la merma del valor del estudio universitario y la dificultad para lograr la inserción en el mercado laboral de estos jóvenes.

Considerando que este trabajo se limita sólo a indagar en una modalidad de arreglo familiar que se observa en este estilo de familia cabe aclarar que éste es sólo uno de los posibles y no es generalizable a otras configuraciones familiares pertenecientes a contextos socioculturales diversos ya que en éstas se observa la presencia de otro tipo de arreglo familiar entre los cuales cabe mencionar por ejemplo: la migración temprana de los adolescentes y los adolescentes que ni estudian ni trabajan incluidos en la categoría "ninis". Estos fenómenos no serán tratados en este escrito debido a que exceden al objetivo propuesto.

Por lo tanto, teniendo en cuenta esta salvedad, en el desarrollo del tema se utilizará la expresión: "estas familias" para hacer referencia a la modalidad de familia (nuclear, de clase media con hijos universitarios en su mayoría del Área Metropolitana de la Ciudad de Buenos Aires) en la cual el fenómeno abordado se presenta.

En estos casos, dicha prolongación implica que la etapa del nido vacío tarda en iniciarse o bien que nunca comienza ya que la permanencia de estos adolescentes en el hogar familiar puede convertirse en una forma de vida anulando la posibilidad de que se produzca la transición a la etapa siguiente.

La dilación excesiva de esta transición retarda la culminación del proceso de diferenciación, el cual supone que el hijo ha logrado no sólo la autonomía física y económica, sino también la adquisición de autoridad personal en el sistema familiar.

¿Qué consecuencias subjetivas tanto en los jóvenes como en los adultos podría traer aparejada esta situación?

Dado que se trata de un fenómeno complejo, el mismo será tratado desde distintos ejes: el contexto socio-cultural actual de este tipo de familias, la institución familia como escenario en el cual el fenómeno citado se inscribe y los atributos de la subjetividad de los jóvenes y adultos del estilo de familia a la cual este trabajo hace referencia, reconociendo que ambos pertenecen a mundos generacionales que poseen características diferentes en lo que refiere a sus metas, valores, estilos de vida e ideales.

Para el abordaje del tema serán tomados en consideración algunos resultados de un incipiente estudio de campo que se realizó en el Instituto de Investigaciones en Ciencias Sociales de la Universidad Argentina de la Empresa durante el año 2013. Dichos resultados se obtuvieron a partir de la toma de 300 entrevistas personalizadas semi-estructuradas a los siguientes grupos etarios: 250 a jóvenes con estudios universitarios en curso y finalizados de entre 18 y 35 años, 50 a padres 
con hijos con las citadas características y 489 encuestas divididas entre: 340 jóvenes de 18 a 35 años y 149 a padres de jóvenes del Área Metropolitana de la Ciudad de Buenos Aires de clase media y media alta.

\section{Desarrollo}

La modalidad de familia que se aborda en este trabajo se inscribe en el contexto de la sociedad posmoderna, la cual se diferencia de la moderna principalmente por el reemplazo de la primacía de la producción y del intercambio de bienes por la primacía del consumo y del intercambio de símbolos. El trabajo en la sociedad capitalista ocupaba el mismo status que hoy ocupa el consumo en la sociedad posmoderna, por lo que los sujetos se convierten en consumidores simbólicos, debido a que es justamente en los símbolos en donde encuentran valor. Los sujetos se implican en el sistema como consumidores más que como productores.

La posmodernidad se caracteriza, además, por una actitud relativista como consecuencia de la caída de los proyectos y utopías que se gestaron en la modernidad, cuya finalidad era darle legitimidad a las instituciones, las políticas sociales y los modos de pensar.

La pérdida de la capacidad instituyente de las instituciones tradicionales dio lugar al surgimiento de una sociedad individualista. Esta individuación implica que los sujetos están condenados a "ser ellos mismos", obligados a construir identidades ya que, a diferencia de la etapa sólida de la modernidad, no vienen dadas de antemano por las instituciones que transitan (Bauman, 2008)

Esto significa que el proceso de individuación conduce al individuo a iniciar una infinita carrera para lograr el reconocimiento social. La identidad se convierte en un proyecto, algo a construir constantemente, no es algo dado. Ésta se construye en el consumo, cada producto de consumo viene con una identidad incluida. El proceso de construirse consiste en: barajar identidades, descartar las ya implementadas y adoptar otras nuevas.

El cuerpo se convierte en portador de los signos que proveen de identidad social. La vestimenta, el calzado, el corte de cabello y el uso de tecnología son ejemplos de estos signos.

Sintetizando esta situación, Bauman (2008) sostiene que el rasgo distintivo de la posmodernidad puede resumirse en la siguiente afirmación: "Consumo, luego existo". Sin embargo si se compara con la enunciación original cartesiana: "Cogito ergo sum: pienso luego existo", se observa una distinción que la diferencia "sustancialmente". Descartes arriba, luego del arduo recorrido iniciado desde la duda metódica, a la intuición: Si pienso tengo que ser algo: una sustancia pensante. Si, justamente, una sustancia ¿Qué se entiende por sustancia? Lo que no cambia, lo permanente, algo que está por debajo de los cambios. Pero en la sociedad posmoderna, la identidad, el resultado del consumo, no es nada cercano a lo permanente, al contrario, es algo que para ser tiene que mutar permanentemente, cambiar, "reciclarse"

Los sujetos no tienen que aferrarse a la identidad, la permanencia se convierte en un disvalor.

Ser libre es desconectarse de lo consumido y consumir "lo nuevo", dejar atrás lo que "se tuvo/fue", para "ser/tener" de una manera novedosa, que también se convertirá próximamente en deshecho. Los sujetos son al mismo tiempo libres y esclavos porque tienen la posibilidad de echar mano a la multiplicidad de identidades que ofrecen los objetos de consumo, pero paradójicamente no pueden detener la carrera, no pueden quedarse con ninguna. Están obligados a seguir eligiendo sino corren 
el riesgo de dejar de ser. Lo permanente ya no es bienvenido, la sustancia cartesiana de esfumó, ahora lo único que queda como permanencia es la obligación de "transformarse"

Por otro lado, si bien el reinado del principio del placer ha destronado al principio de realidad, esta forma de vida no está exenta de sinsabores, el consumismo trae aparejado la insatisfacción eterna acompañada por miedos, desconcierto y desesperación.

Este estado de renovación permanente sitúa a los sujetos exclusivamente en el aquí y ahora. No hay inversión en proyectos a largo plazo porque distraen la atención del proceso de "autofabricación" (Bauman, 2008). Entonces, el futuro se vuelve incierto.

Estos conocimientos imperantes en la actualidad en lo que refiere a definir cómo ser, como orientar y que sentido darle a la vida atraviesan toda la trama de instituciones. (Burr, 1995; Gergen, 1993)

Cabe preguntarse entonces de qué manera impacta la sociedad posmoderna en el estilo de familia en la cual se produce el fenómeno que se busca explicar: "nido lleno".

Antes de abordar este interrogante será necesario caracterizar este tipo de familia, recordando que sus atributos no son generalizables a otras configuraciones familiares.

En tanto institución, esta modalidad de familia ha estado expuesta a múltiples transformaciones según época y cultura, pero aún así mantiene su identidad a través del desempeño de sus funciones, las cuales definen su carácter.

Estas son: reproducción, socialización, crianza de los hijos, cuidado y protección de los miembros que la componen, regulación de la vida sexual e integración a la sociedad como miembros plenos. Además, es la encargada de fijar aspiraciones, valores y motivaciones de los individuos y es responsable en gran parte de su estabilidad emocional, tanto en la infancia como en la vida adulta.

Flaquer (citado en Gracia Fuster y Musitu Ochoa, 2000, p.53) sintetiza las funciones que competen a esta modalidad de institución familiar:

El grupo familiar se constituye como agregado de ocio y consumo, de plataforma de ubicación social, de núcleo de relación social, de palanca para la constitución del patrimonio, de cauce para hallar empleo, de punto de apoyo y de recursos de amparo en caso de crisis y de unidad de prestación de cuidados asistenciales y de salud.

Por lo tanto, estas familias se organizan para cumplir dos funciones básicas: el desarrollo personal de los hijos y la socialización.

Es decir, las tareas básicas de dichas familias van más allá de la crianza de los hijos, entendida como alimentación y salud física, e incluso más allá de la protección y el afecto, pues se busca el desarrollo personal de todos sus miembros, niños, adultos y ancianos, y su incorporación activa al entorno social.

Estas familias, además, tienen como meta mantener la propia identidad y la cohesión familiar (Gimeno, 1999).

Entonces, esta forma de familia si bien presenta continuidades representadas por sus funciones, se encuentra ligada a los procesos de transformación de la cultura contemporánea. 
Como parte de los diferentes procesos históricos, la familia (refiriéndose a esta modalidad específica de familia), no es ni un receptor pasivo de los cambios sociales ni el elemento inmutable de un mundo en constante transformación (Gracias Fuster y Musitu Ochoa, 2000, p.44).

Por consiguiente, este estilo de familia es una institución en la cual se sintetizan las nuevas expresiones de la cuestión social desde lo objetivo, que se plasma en las nuevas configuraciones que adquiere y en lo subjetivo respecto de la identidad de sus miembros.

Es por ello que al hacer referencia a este modo de familia se entiende que el significado de ésta no pasa por su conformación, ni por la permanencia de sus integrantes dentro de un ciclo determinado, con tramas de relaciones claramente definidas y roles construidos en forma armónica, sino que constituye un espacio de construcción y reconstrucción permanente, en el cual se cruzan intereses e ideales diversos que reclaman su reconocimiento. (Carballeda, 2011).

Siguiendo estas reflexiones en torno a esta modalidad de familia en tanto producto cultural y por ende sujeta a múltiples cambios sobre un trasfondo de continuidad, cabe preguntarse: ¿Cuáles han sido las transformaciones que ha sufrido esta institución en los últimos años?

Algunas de ellas son: las parejas se casan más mayores, la edad de la maternidad se retrasa, la tasa de natalidad disminuye, se comparten las tareas domésticas y se distribuye entre ambos cónyuges las responsabilidades del aporte económico, aumenta la participación de la mujer en el mercado laboral, y se incrementa la tasa de divorcio, separación y de la monogamia secuencial o serial.

Entre los factores que repercutieron en esta vida familiar cabe mencionar: la migración de las familias hacia las grandes urbes en búsqueda de fuentes de trabajo, el control de la fecundidad natural y fecundidad asistida y el fin del amor religioso y románico. (Wainstein, 2012)

A estas modificaciones se agrega el aumento de la esperanza de vida que acarrea el fenómeno de la verticalización de dichas familias (muchas generaciones con pocos miembros), el proceso de socialización invertida, el surgimiento de la generación "sándwich" (adultos que están en medio de sus padres e hijos demandantes) y el fenómeno "tupac amarú" (tironeo por parte de los adultos mayores y por parte de los adolescentes tardíos que siguen reclamando dedicación) (Acrich de Gutmann, 2010)

El fenómeno que se aborda en este trabajo se incluye, dentro de los cambios acontecidos en el seno de las familias nucleares de clase media y media alta con hijos en su mayoría universitarios: los hijos jóvenes adultos permanecen más tiempo en la familia de origen, se prolonga el inicio de la etapa de nido vacío. ${ }^{2}$

Moreschi (2007) afirma que en estos casos, las familias, en lugar de dar inicio a otra etapa, como

\footnotetext{
${ }^{2}$ El estudio de campo realizado refleja los siguientes datos con respecto a este fenómeno: el 78,6\% de los jóvenes menores de 25 años viven con sus padres. Este porcentaje se reduce al $51,4 \%$ para los de 25 o más años, es decir que aún en este grupo etario, la mayoría continúa viviendo con sus padres. Los hogares donde viven estos jóvenes, en general tienen entre 4 y 5 miembros en promedio. Más de un $40 \%$ de los jóvenes, independientemente de la edad, manifiesta que le gustaría irse de la casa de sus padres porque le daría más independencia. Dentro de los menores de 25 años, algunos dicen que cuando sean más grandes $(27,5 \%)$ y otros que no por ahora (22,7\%). Dentro de los de 25 y más años, un $20,5 \%$ también manifiesta que no por ahora y un $17,1 \%$ que lo antes posible. Es de notar que la incidencia de quienes responden que por ahora no se quieren ir porque están cómodos, es más alta entre los de 25 y más años, que entre los más chicos (7,5\% vs, 4,5\%).
} 
ocurre con cualquier entidad vital, se estancan en una de ellas deteniendo su evolución y lo que debería ser una etapa a transitar y superar se convierte en una forma de vida.

Además, la edad se vuelve irrelevante con relación a la realización de tareas demandadas por cada sociedad, por ejemplo, si antes se tenía como expectativa que los jóvenes adultos se separaran de la convivencia familiar alrededor de los 21 años, ahora la edad deja de ser significativa, puede ser a los 25 , pero también a los 30,35 o incluso más.

Como consecuencia de esta modificación el comienzo de la adultez, según la OMS (Organización Mundial de la Salud) se ha corrido de los 21 a los 25 años, surgiendo así una nueva categoría denominada adolescencia prolongada o tardía que es aquella que supera los 25 y a veces también los 30 años.

Gimeno Collado (1999, p.38) afirma que "la separación de los hijos del hogar familiar sólo se ve justificada por la emancipación de los mismos, lo que se da prácticamente cuando han encontrado pareja estable y han llegado a ser económicamente autosuficiente".

Este proceso, en estas configuraciones familiares, se ha desplazado en los últimos años alrededor de 10 años o más.

Estas familias se han encontrado, entonces, con el problema de extender en el tiempo las funciones de integración de sus miembros a la sociedad, punto de apoyo y palanca para la constitución del patrimonio de las jóvenes generaciones. Es decir, se retarda el inicio de la etapa de nido vacío, lo cual trae aparejada la dilación de la autonomía de los hijos.

Willianmson y Bray (citados en Gimeno Collado, 1999, p.114) sostienen que:

La emancipación del hijo implica que este deja de necesitar el cuidado parental y puede iniciar con sus padres una relación de iguales, sin dependencias; los ve como seres humanos y los acepta como son, como consecuencias de su propio cambio: de la clarificación de su propio self.

Es indudable que este estilo de familia se encuentra en un proceso de transformación permanente, lo que ha dado lugar al surgimiento de nuevos fenómenos en su seno, como el fenómeno de interés en este trabajo.

Para poder comprender estos cambios será fundamental indagar en la otra dimensión de análisis que se ha propuesto: ¿Cómo son los jóvenes que habitan estas familias? ¿Qué valor le dan al logro de su autonomía?

En primer lugar, dado que se hace referencia a estos jóvenes como adolescentes eternos o tardíos cabe precisar la distinción entre adolescencia y adolescencia tardía para luego centrarse en las preguntas precedentes.

El término adolescencia proviene, etimológicamente, del verbo latino adolecere cuyo significado es: crecer. Muchas veces se asocia con adolecer, que significa carencia, falta. Sin embargo, estos dos significados señalan aspectos del mismo fenómeno: ambos apuntan al proceso de constitución de la persona adulta, por un lado alude a la carencia en tanto inmadurez y por otro al crecimiento en tanto potencia.

Si bien los límites de la adolescencia no son precisos ya que estos dependerán del contexto cultural 
y familiar en la cual se desarrolla y de la historia singular de cada individuo que la cursa, su rasgo distintivo es que en dicha etapa se inicia el proceso de construcción del relato personal y se significan y valoran los proyectos propios.

Se habla de adolescencia prolongada cuando la resolución del conflicto adolescente se demora y por lo tanto es tardía también la consolidación de la identidad.

Si bien en algunos casos, tal como se mencionaba previamente, se trata de una postergación, en otros a pesar de tener la edad suficiente para abandonar "el nido" continúan en él, convirtiéndose en un "estilo de vida" que constituye el fenómeno denominado "nido lleno".

Aclarado este punto, se retoma la pregunta acerca de estos jóvenes y el valor que le dan a su autonomía.

Los jóvenes a los que se hace referencia nacieron entre los años 80 y 90, por lo que se trata de una generación educada en la sociedad digital.

Feixa (2006) sostiene que el escritor Douglas Coupland ya había enunciado el término "Generación X" para referirse a una juventud marcada por las incertidumbres y las paradojas de la sociedad postmoderna, y además por la falta de un sistema de valores sólido. Sin embargo, según Feixa (2006), la característica que distingue a los jóvenes y las jóvenes de fin de siglo es su acceso a las nuevas tecnologías de la información y la comunicación, sobre todo su acceso a la red, por lo que la denomina "Generación R (red)".

Sus modos de consumo han hecho que el sector tecnológico se desarrolle y sus relaciones se encuentran mediatizadas por la tecnología.

La práctica del consumo se convierte, para ellos, en la vía para acceder a la estima social, carrera infinita y muy costosa. Si cuentan con recursos originados por sus propios trabajos o si son brindados por sus padres, los destinan a "construir sus identidades" por medio de la adquisición de objetos.

El hogar familiar se convierte, para ellos, en la base firme que asegura que la carrera de seguir consumiendo puede continuar. En medio de este panorama, en el cual la contingencia y el presente prevalecen sobre el futuro, el proyecto de lograr autonomía económica se desdibuja, pierde interés, más aún se presenta como un sin sentido.

Uno de los mensajes que reciben los jóvenes es: aprovechá el presente, disfrutá, sé feliz [...] Estos mensajes son una de las causas por las cuales el joven no puede poner su interés en la consolidación de su independencia. ¿Cómo trabajar para pagar el alquiler, hacerse cargo de la comida, ropa y además tener tiempo y dinero para salir, beber tragos, viajar y comprar objetos de marca? Algo habrá que resignar y ese algo es la independencia. (Moreschi, 2007, p.106)3

Otro aspecto del contexto que impacta en el proceso de emancipación de estos jóvenes, consiste en los efectos que ha producido la globalización en distintos ámbitos, pero fundamentalmente en el económico, trayendo como consecuencia no sólo altos niveles de desocupación sino también retraso en la inserción laboral de jóvenes que han finalizado la etapa de formación. ${ }^{3}$

Esta demora luego del período formativo aumentó las inseguridades propias de la adolescencia por

3 Según los resultados de la muestra, los jóvenes menores de 25 años manifiestan que los padres debieran ayudar a los hijos económicamente hasta que consigan un trabajo (34,2\%) seguido de "hasta los 21 años" (26,3\%). Dentro del grupo de "25 y más años", el 29,3\% manifiesta que deben ayudarlos hasta que terminen la carrera y un $25,9 \%$ manifiesta hasta que consigan un trabajo. 
lo que a estos les resulta más cómodo y tentador quedarse en la casa paterna que enfrentarse a un desafío para el que no se sienten preparados y que tampoco promete ventaja alguna. Es así como la satisfacción por la independencia no resulta un estímulo motivador. ${ }^{4}$

Otra situación a considerar es el lugar que tienen las instituciones educativas actualmente, en comparación con el que ocuparon históricamente en la sociedad.

En la modernidad la estadía en las instituciones educativas apuntaba a la construcción de la conciencia de trabajo y del esfuerzo en el presente para un futuro prometedor.

Hoy la acción educativa es despojada de sentido, lo que conduce a la construcción del espacio educativo como un "no lugar", es decir, como un espacio por el cual "se pasa de largo", como si fuera un tránsito sin destino final y cuyo valor no es otro más que ese.

La universidad, incluso, se ha convertido en un paso transitorio para poder acceder a estudios superiores, de nivel de postgrado, ya que "con el título en la mano no basta". (Formichelli, 2011)

A esto se agrega que en muchos casos la formación universitaria no tiene por objeto la aplicación práctica laboral o de bregar por su propio sustento sino que se constituye en una forma de vida, es decir, el estudio se convierte en una manera de seguir dependiendo de los padres, en la medida que son los adultos los que pagan por ellos.

Además el modelo de adulto con el que cuentan estos jóvenes se presenta poco motivador. Se encuentran con un adulto cargado de responsabilidades y compromisos.

Frente a un modelo que no seduce, el joven se convierte en víctima de un consumo que viene a sustituir ese vacío, esa pérdida de sentido.

En síntesis, este joven se siente poco motivado para adquirir autonomía y avocarse a un proyecto a largo plazo, por un lado porque el discurso dominante de la sociedad consumista le muestra como único camino para obtener reconocimiento social el consumo inmediato, y por el otro porque el modelo del adulto con el que cuenta no le resulta tentador.

Es en este punto donde se abre la otra dimensión a indagar: ¿Cómo son los padres de estos adolescentes? ¿Cuáles son sus proyectos?

Estos padres nacieron entre las décadas del 50 y 60 .

Feixa (2006) propone, para esta generación, siguiendo a diversos autores, la denominación "Generación bc (before computer), para distinguirla de la "Generación ac (after computer)" en la cual se sitúan sus hijos.

La primera, se enfrentó con el derrumbe de las utopías y con la ausencia de otra trama de significados que diera sentido a sus vidas. Por este motivo acaba siendo incluida en un mundo contingente y consumista, en el que coexisten en tensión relatos antiguos, producto de prácticas ya en desuso con otros que responden a las nuevas conductas.

\footnotetext{
${ }^{4}$ A partir de los resultados del trabajo de campo, se observa que del total de jóvenes que no trabajan, un $63 \%$ en promedio argumenta que es por decisión propia. En los menores de 25 años, esta cifra se eleva al $87,5 \%$, en tanto que desciende a un $53,5 \%$ en aquellos de 25 años o más. En éstos últimos un 34,1\% argumenta a su vez no conseguir trabajo. Los que no trabajan obtienen sus ingresos en mayoría de sus padres ( $77 \%)$, el resto de ahorros propios $(11 \%)$, alquileres $(5 \%)$ u otras fuentes $(7 \%)$.
} 
Estos habitaron un mundo moderno en el cual se valoraba el proyecto a largo plazo, donde el futuro era previsible ya que el plan de vida tenía como finalidad la trascendencia.

Se muestran disconformes con sus vidas, sienten que "no encajan" en la sociedad actual. El modelo de madurez que muestran es poco tentador y lo único que ven como permanente son las obligaciones.

Entonces, ¿Cuáles son sus proyectos hoy en día?

Dado que estos padres se encuentran en una cultura donde el lugar para sus sueños casi ha desaparecido, encuentran en el proyecto del hijo una manera de redireccionar los propios anhelos, que se han tornado inalcanzables. Así, la prolongación de la adolescencia de sus hijos se convierte en una estrategia para postergar el monótono destino que la falta de proyectos propios les depara.

Los hijos se transforman en el motivo de sus existencias, ponen toda su energía al servicio de ellos, financian el proyecto de los jóvenes y en algunos casos, incluso, lo diseñan conforme a sus propios sueños.

Esta situación implica un costo alto para los hijos quienes terminan recluidos en un reinado de límites estrechos, la familia, resignando el desarrollo personal que significa hacerse un lugar en el mundo (Moreschi, 2007)

Esta situación no es sin conflicto, ya que si bien para los padres proyectar sus metas personales en los hijos resulta una manera de llenar un vacío de sentido, esta tarea les resulta agotadora e interminable.

Generalmente, en el abordaje de esta problemática, se ha puesto la mirada en los motivos que llevan a los hijos a permanecer en la familia de origen indagando sobre las posibles causas que llevan a que esta situación se produzca. Sin embargo, pocas veces se ha puesto el foco en los motivos que llevan a los padres a responder a los requerimientos de los hijos.

Si bien, las quejas de estos padres se hacen escuchar y en muchos casos a esto se le suma la emergencia de una amplia variedad de síntomas tales como: cefaleas, gastritis, colesterol alto, úlceras, éstos se convierten en cómplices de la "comodidad" de sus hijos porque tal vez ésta sea la manera en la que encuentren un consuelo frente a sus sueños perdidos.

Los intereses de los hijos son inagotables, dado que pertenecen a la cultura posmoderna, en la cual hay que estar siempre renovándose, consumiendo. Los padres suponen que en algún momento esta carrera terminará y los hijos se convertirán en lo que ellos mismos han soñado. Pero esto no es así, corren por veredas distintas, mientras los padres imaginan que el hijo armará su proyecto a futuro estudiando una carrera universitaria, por ejemplo, los hijos ven en la carrera universitaria solo un lugar de paso, una marca de consumo más, nunca la última. Luego le sigue la formación de posgrado, un viaje, más tecnología. Es por ello que esta situación parecería no terminar nunca.

Sin embargo, para estos padres no es fácil ponerle fin, tal vez porque se quedarían sin ilusiones.

Algunos padres, sostiene Moreschi (2007), crían a sus hijos en un plano de igualdad, se vuelven permisivos y justifican su dificultad para poner límites como amplitud o libertad.

Esta presencia de límites difusos parece sostener la ilusión de estos padres de eterna juventud, condición altamente valorada en la cultura posmoderna, al mismo tiempo que evita que los hijos se independicen de ellos y como consecuencia, quedarse sin proyectos. 
El nido lleno los agota, pero también temen que quede vacío. Si los hijos logran su autonomía económica y se van, los padres se quedan sin proyectos y la ilusión de eterna juventud, sostenida ilusoriamente en la creencia de que sus hijos siempre dependen de ellos porque son adolescentes (eternos), se desmorona.

Es decir, el "nido lleno" también constituye para estos adultos no sólo un reaseguro contra la soledad que otrora representaba el "nido vacío" sino también un terreno fértil para la búsqueda de la consecución de sus propios ideales: ¿ideales inconclusos, postergados, pasados de moda? Estos padres se debaten entre dos anhelos que entran en conflicto: por un lado el de que los hijos sigan siendo "adolescentes eternos" y por otro el de ayudarlos a crecer y "pasarles la posta". 5

Como resultado del recorrido precedente se observa que la subjetividad de estos jóvenes difiere de la de los adultos en cuanto a sus metas, proyectos e ideales.

Cabe preguntarse de que manera estás diferencias impactan en las etapas normativas del ciclo de vida de este estilo de familia.

Cada etapa del ciclo de vida familiar se define por el modo en que cada familia logra el desarrollo personal de sus miembros, su integración activa a la comunidad y mantenimiento de la cohesión familiar que son sus funciones básicas.

Para este fin las familias desarrollan, en cada etapa, un modo particular de atender a sus funciones utilizando los recursos con los que cuenta o bien desarrollando nuevos.

Cada cultura y cada época establece los roles, rasgos, tareas y parámetros específicos de cada una de las etapas y establecen los límites para que se resuelvan las tareas en cada núcleo familiar.

"La funcionalidad y satisfacción familiar depende, desde un punto de vista objetivo, del logro de estas tareas de etapa y desde el punto de vista subjetivo de la proximidad entre los logros y las expectativas familiares" (Gimeno, 1999, p.49).

En el contexto actual se observa que este tipo de familia se encuentra extendiendo en el tiempo la función de sostén de la etapa de emancipación de los hijos del ciclo de vida familiar. Por un lado, para saberse que siguen cumpliendo con uno de los objetivos más tangibles de la misma que es la educación de sus hijos y sentirse realizados, y por el otro para perpetuar una forma de vida familiar que no enfrente a sus miembros adultos con el duelo por lo no logrado, ni a los jóvenes con una realidad que promete un desafío para el cual sienten no contar con recursos y que tampoco asegura ventaja alguna.

Este conflicto, producido tanto por contradicciones en el seno de la familia como por aquellas que provienen del contexto sociocultural, se encuentra en la base de la prolongación del proceso de emancipación de estos jóvenes.

La emancipación de los hijos del hogar constituye una de las transiciones de la vida familiar. Sin

\footnotetext{
5 Los resultados del trabajo de campo muestran que entre las formas de ayudar, el $57,4 \%$ del total de los padres que dan ayuda a sus hijos, le dieron una extensión de la tarjeta de crédito, y un $25 \%$ del total les dan una mensualidad fija (el $75 \%$ no da mensualidad fija). Eso implica que un $16 \%$ del total de padres que ayudan les dan ambas cosas, extensión de tarjeta y también mensualidad fija.

Los padres también pagan algunos gastos con diferentes prioridades. En el grupo más prioritario están los Alimentos e Internet en casa, le siguen medicina prepaga y abono del celular", y luego, la cuota de la universidad, otros gastos para el estudio, ropa y el aparato del celular.

Cabe destacar que el $80 \%$ de los padres que ayudan económicamente a sus hijos siente que ellos los retribuyen afectivamente.
} 
embargo, en la actualidad se replica en las familias a las que se hace referencia cierta "fijación" o "estancamiento", en esta etapa. Esto significa que estas familias prolongan la realización de tareas de etapas anteriores, lo que representa un impedimento del progreso hacia modelos de organización que se ajusten mejor a las demandas evolutivas de sus miembros.

Este estancamiento consiste en la rigidez del sistema familiar que no permite el movimiento de sus miembros. Esa modalidad de funcionamiento familiar constituye uno de los arreglos posibles en un contexto sociocultural que ha dejado a los adultos sin poder consolidar sus proyectos personales y a los jóvenes sin proyectos a largo plazo y poco interés en el logro de su autonomía. Como consecuencia, los hijos no se separan del núcleo familiar de origen y la consolidación de la identidad personal se demora o bien se obstaculiza si la situación se transforma en una forma de vida.

Cabe preguntarse si esta situación significa realmente una transición o si se ha tornado una forma de vida.

\section{Conclusión}

"La familia posmoderna se caracteriza por ser diversa, fluida e inestable y por la conjunción de lo viejo y lo nuevo" (Roigé, 2006, p.36).

En estas familias coexisten dos culturas. Por un lado la de los padres que siguen manteniendo las creencias y valores de la modernidad: el futuro, el esfuerzo, la responsabilidad, y por otro lado la de los hijos, que viven en un mundo de acción, placer, inmediatez.

Estas familias se encuentran viviendo entre valores opuestos y mensajes contradictorios.

Estos padres sostienen económicamente a hijos que ya tienen edad para ser autónomos económicamente, pero no sólo lo hacen por sus hijos, para brindarles el sostén que como padres sienten que deben proveerles, sino también por ellos, para seguir teniendo un proyecto a futuro que de sentido a sus vidas.

Los hijos, en cambio, tienen claro que hoy el estatus está dado por el consumo. El mensaje social es: "dime qué consumes y te diré quien eres". Para ellos el título puede servir para obtener una marca más de consumo entre otras, o bien para asegurarse la provisión de dinero por parte de sus padres para estudiar, mientras ellos siguen consumiendo bienes que les aseguran el reconocimiento social.

Es por ello que aunque estos jóvenes trabajen y ganen dinero como para poder irse de la casa de sus padres, más de la mitad del ingreso lo gastan en bienes de consumo personal, "gustos" (ropa, tecnología, salidas). Ni los padres ni los hijos consideran que estos últimos deberían renunciar a ciertas comodidades, hacer determinados sacrificios.

Estos jóvenes no parecen encontrar en esta situación incomodidad alguna, es más, plantean todo lo contrario, se sienten "muy cómodos" y justifican el aporte de los padres argumentando que se trata de una función inherente e ineludible que compete al rol de adulto.

Es indudable que estos hijos encuentran en el "nido" un ámbito que les brinda sostén para la realización de sus metas instantáneas, pero no sólo son los hijos los que encuentran un espacio propicio para tal fin, sino que los padres también aunque sus metas sean cualitativamente diferentes. En síntesis, esta modalidad de familia está en transformación permanente; hoy uno de sus cambios 
es la extensión en el tiempo de la etapa de proceso de emancipación de los hijos del hogar. Esto se explica por la confluencia de diversos ideales en el seno de la familia. Por un lado los padres sostienen a sus hijos para darle sentido a sus propias vidas, por otro lado los hijos, buscan construir su identidad personal a través del consumo, lo cual se torna contradictorio con el diseño de un proyecto a futuro que les permitiría el logro de la autonomía económica. Por tal motivo para poder seguir consumiendo se apoyan en la ayuda de los padres.

El nido es un "nido lleno de ideales", ideales "multicolores", diversos, que conforman una nueva receta apta para el "consumo" de toda la familia. Siguiendo con la metáfora de la comida familiar, en el slogan se agrega: "les va a "encantar a todos"

Así mismo, las contradicciones no sólo se encuentran en el seno de estas familias, sino que también se encuentran en la sociedad misma. Se trata de una sociedad en la cual estos adultos no encuentran un terreno fértil para la consecución de sus propios proyectos y que promueve en estos jóvenes más que la búsqueda de un proyecto a largo plazo, el valor por el placer inmediato. A esto se agrega que si bien en ésta se valora "lo joven" por sobre "lo viejo" no ofrece ventaja alguna en lo que refiere a la inserción laboral de ellos y que la formación universitaria tampoco asegura el reconocimiento social.

¿El fenómeno del nido lleno es una transición que tendrá su término o se ha conformado en una transición interminable, es decir un estilo de vida para estas familias?

Si este "tiempo de espera" en esta etapa del ciclo de vida familiar se vuelve "interminable" ¿puede convertirse en una amenaza contra el desarrollo personal de los hijos representado por la autonomía económica?

\section{Referencias bibliográficas}

Acrich de Gutmann, L (2010) Consideraciones sobre familia y envejecimiento. Trabajo inédito, material del Programa de Actualización en Psicogerontología, UBA.

Bauman, Z. (2008) Amor líquido: acerca de la fragilidad de los vínculos humanos (1 $1^{\mathrm{a}} \mathrm{Ed} .10^{\mathrm{a}}$ Reimp.) Buenos Aires: Fondo de Cultura Económica.

Bauman, Z. (2008) Vida de consumo (1 ${ }^{\text {a }}$ Ed. $1^{\text {a }}$ Reimp.) Buenos Aires: Fondo de Cultura Económica.

Burr, V. (1995). An Introduction to Social Constructionism. London: Routledge (traducción).

Carballeda, A.J.M. (2011) Algunos cambios en la esfera de la familia. Una mirada desde la intervención en lo social. En Elías, M.F. (Comp) Nuevas Formas familiares. Modelos, prácticas, registros. Buenos Aires: Espacio Editorial.

Feixa, C. (2006). Generación XX. Teorías sobre la juventud en la era contemporánea. Revista Latinoamericana de Ciencias Sociales, Niñez y Juventud, 4(2), 3-18.

Formichelli, M. (2011) Paradojas de la juventud posmoderna. De las categorías teóricas a la realidad. En Villa, A. Infantino, J. y Castro, G. (comps) Culturas juveniles. Disputas entre representaciones hegemónicas y prácticas. Buenos Aires: Noveduc. 
Gergen Kenneth J. (1993) El movimiento del construccionismo social en la psicología moderna. Rev. Sistemas Familiares, 9, 2, agosto. Buenos Aires.

Gimeno, A (1999) La familia: el desafío de la diversidad. Barcelona: Ariel.

Gracia Fuster, E, Musitu Ochoa, G. (2000) Psicología social de la familia. Barcelona: Paidós.

Moreschi, G. (2007) Adolescentes eternos: ¿por qué nunca se van de casa? (1ª ed.) Buenos Aires: Paidós.

Roigé, X. (2006) Familias de ayer, familias de hoy: cambios y continuidades en Cataluña, Barcelona: Icaria Editorial S.A.

Wainstein, M. (2012) Familia, terapia y postmodernidad. Revista Intersecciones, 2(4) Disponible en http://intersecciones.psi.uba.ar/index.php?option=com content\&view=article\&id=126:familiaterapia-y-posmodernidad\&catid=9:perspectivas\&ltemid=1 